\title{
Time points for obtaining representative values of 24-hour blood pressure in chronic kidney disease
}

Jiwon Ryu ${ }^{1}$, Ran-hui Cha ${ }^{2}$, Dong Ki Kim³ ${ }^{3}$ Ju Hyun Lee ${ }^{4}$, Sun Ae Yoon ${ }^{5}$, Dong Ryeol Ryu ${ }^{6}$, Jieun Oh ${ }^{1}$, Sejoong Kim ${ }^{4,7}$, Sang-Youb $\mathrm{Han}^{8}$, Eun Young Lee ${ }^{3}$, and Yon Su Kim ${ }^{9}$

\begin{abstract}
${ }^{1}$ Department of Internal Medicine, Hallym University College of Medicine, Chuncheon; ${ }^{2}$ Department of Internal Medicine, National Medical Center, Seoul; ${ }^{3}$ Department of Internal Medicine, Soonchunhyang University College of Medicine, Cheonan; ${ }^{4}$ Medical Research Collaborating Center, Seoul National University Bundang Hospital, Seongnam; ${ }^{5}$ Department of Internal Medicine, College of Medicine, Uijeongbu St. Mary's Hospital, The Catholic University of Korea, Uijeongbu; ${ }^{6}$ Department of Internal Medicine, Ewha Womans University School of Medicine, Seoul, Korea; ${ }^{7}$ Department of Biomedical Engineering, University of Michigan, Ann Arbor, MI, USA; ${ }^{8}$ Department of Internal Medicine, Inje University College of Medicine, Goyang; ${ }^{9}$ Department of Internal Medicine, Seoul National University College of Medicine, Seoul, Korea
\end{abstract}

\section{Received: April 20, 2014 \\ Revised : July 10, 2014 \\ Accepted: July 31, 2014}

\author{
Correspondence to \\ Sejoong Kim, M.D. \\ Department of Internal \\ Medicine, Seoul National \\ University Bundang Hospital, 82 \\ Gumi-ro 173beon-gil, Bundang-gu, \\ Seongnam 13620 , Korea \\ Tel: $+82-31-787-7051$ \\ Fax: $+82-31-787-4052$ \\ E-mail: sejoong2@snu.ac.kr
}

Background/Aims: Ambulatory blood pressure (BP) monitoring has been widely recommended for evaluating the status of $\mathrm{BP}$, but is lacking in practicality. Determination of the specific time points for BP measurement that are representative of 24-hour mean BP could be useful and convenient in hypertensive patients with chronic kidney disease (CKD).

Methods: A total of 1,317 patients for whom 24-hour ambulatory BP monitoring was performed were enrolled in a multicenter study on hypertensive CKD. We analyzed the time points at which systolic blood pressure (SBP) values exhibited the smallest differences from 24-hour mean SBP (mSBP). We included office mSBP and analyzed the relationships between SBPs at the office and the time points with the smallest differences from 24-hour mSBP using several methods.

Results: The time points with the smallest differences from 24-hour mSBP were 7:00 AM, 2:00 PM, and 9:30 PM. In regression analysis, SBPs at 7:00 AM and 9:30 PM were better correlated with 24-hour mSBP than SBPs at 2:00 PM and the office. The proportions of patients with SBPs within $30 \%$ of 24 -hour mSBP were higher at 7:00 AM and 9:30 PM. The best consistency between the uncontrolled hypertensive groups, defined as $\geq 135 \mathrm{mmHg}$ of 24 -hour $\mathrm{mSBP}$ and higher values of SBPs corresponding to $135 \mathrm{mmHg}$ of 24 -hour $\mathrm{mSBP}$, were observed at the 7:00 AM and 9:30 PM time points.

Conclusions: The specific time points for SBPs that correlated well with 24-hour $\mathrm{mSBP}$ in hypertensive CKD patients were 7:00 AM and 9:30 PM.

Keywords: Ambulatory blood pressure measurement; Blood pressure measurement; Chronic kidney disease; Hypertension; Timing for representative blood pressure

\section{INTRODUCTION}

Blood pressure (BP) is a predictor of cardiovascular and cerebrovascular diseases. Furthermore, high BP in chronic kidney disease (CKD) patients is a strong predictor of end-stage renal disease (ESRD) or death [1-4]. Korean data 
for a recent 15-year period (from 1985 to 2009) showed that hypertension is a major cause of ESRD, and the prevalence of hypertension would be increased by CKD itself [5]. Although the gold standard for diagnosis of high BP is still office BP, it has been suggested that out-of-office BP measurements could provide more information, especially given the "white coat effect," masked hypertension, or the dipping pattern. Out-of-office BP measurement methods include ambulatory blood pressure monitoring (ABPM) and home blood pressure monitoring (HBPM) [6]. However, ABPM tends to be inconvenient from a practical viewpoint, whereas HBPM is relatively easy to check and is reproducible [7-9]. Although several studies have suggested the usefulness of HBPM, few have recommended specific time points for HBPM measurements; some studies suggested that BP measurements should be performed more than twice during the day, on several consecutive days, and during the morning and night, but these studies did not provide specific time points for measurements [10-12]. The Japanese Society of Hypertension provided more detailed suggestions-they recommend that $\mathrm{BP}$ should be measured within 1 hour after waking up and just before going to bed, but no specific time points for BP measurement were provided [13]. We considered that a specific time point for BP measurement, which is representative of the 24 -hour mean BP, would be clinically useful for self-management of hypertension. This study was performed to determine a specific time point for measurement of systolic blood pressure (SBP) that is well correlated with the 24-hour mean SBP (mSBP) using a cross-sectional multicenter study involving hypertensive CKD patients in Korea (APrODiTe study) [14].

\section{METHODS}

\section{Study design}

The APrODiTe study assessed BP control and target organ damage (TOD) in hypertensive CKD patients. This study, in which 21 centers participated, was designed as a nationwide, prospective, cross-sectional study performed from October 2009 to May 2011. The Institutional Review Boards of all participating centers approved the protocol and all investigations were performed in accordance with the Declaration of Helsinki. Written informed consent was obtained from all participants. We used data from the APrODiTe study, including BP data, drugs, and laboratory data.

\section{Study population}

We included 1,317 patients who: (1) provided informed consent; (2) were 20 to 75 years of age; (3) had received a diagnosis of hypertension for $>6$ months (or $\geq 3$ clinic visits) prior to study participation and had taken antihypertensive drugs for $>3$ months; (4) had estimated glomerular filtration rate between 15 and $89 \mathrm{~mL} / \mathrm{min} / 1.73$ $\mathrm{m}^{2}$; and (5) complied well with medication and had no prescription changes in the 2 weeks prior to study participation. Patients with the following characteristics were excluded: (1) a prescription change according to ABPM; (2) a history of acute kidney injury or hospitalization; (3) proteinuria $>6 \mathrm{~g} /$ day (spot urine protein/creatinine ratio > 6.0); (4) ESRD on dialysis or a history of kidney transplant; (5) a history of diseases, such as uncontrolled arrhythmia, uncontrolled bronchial asthma/ chronic obstructive pulmonary disease, or primary endocrine diseases, with the exception of diabetes mellitus; (6) pregnant or lactating; (7) nightshift workers; (8) enrollment in other clinical studies within 2 months; or (9) who were deemed inappropriate for participation according to their physician.

\section{BP measurement}

All of the participants were asked to rest in a seated position for 5 minutes and to not drink coffee or smoke for at least 30 minutes before each BP measurement. Experienced staff checked the BP using an Omaron IA-2 automatic BP device (IntelliSens, Omron Corp., Kyoto, Japan) in triplicate at intervals of 1 to 2 minutes and recorded the mean of the final two readings. The ABPM was checked with TM-2430 (A\&D Co., Ltd., Seoul, Korea) every 30 minutes for 24 hours. The ambulatory BP records were considered to be adequate if the monitor had been worn for 24 hours and if there were $\geq 16$ acceptable readings between 8:00 AM and 10:00 PM (daytime) and $\geq 12$ acceptable readings between 10:00 PM and 8:00 AM (nighttime) [15]. We used only SBP in this study, because SBP is considered more useful and important than diastolic blood pressure (DBP) for the diagnosis of hypertension and its relationship with TOD $[16,17]$. 


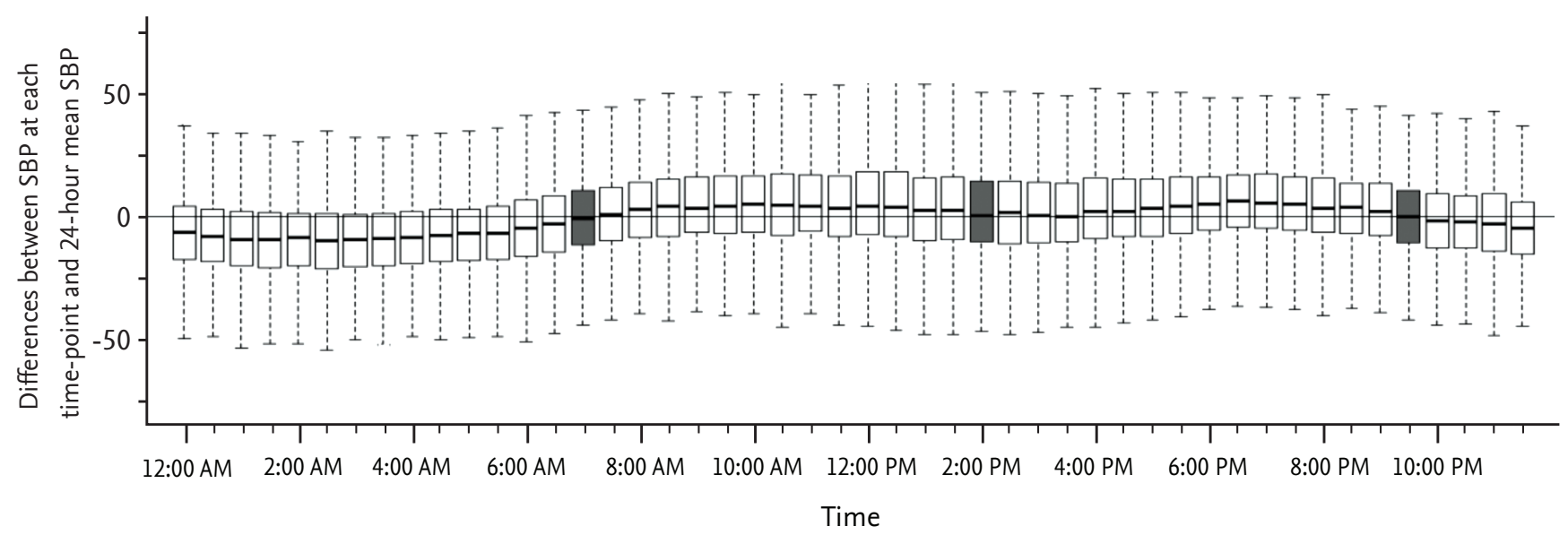

Figure 1. Differences between systolic blood pressure (SBP) values at each time point and the 24-hour mean SBP.

\section{Statistical analysis}

First, we analyzed the differences between the SBPs at each time point and the 24-hour mSBP (Fig. 1). The box plot of this figure indicates the range of $25 \%$ to $75 \%$ of SBP, and the middle line of each box plot indicates the median SBP value at each time point. If the difference at a certain time point was near zero, the SBP at that time point was closest to the 24-hour mSBP, based on the Bland-Altman method [18]. We added office mSBP to the analysis to compare the usefulness of office SBP with the SBP at the selected time points. We evaluated the significance of the differences using Student paired $t$ test inversely; the smallest $t$ value was considered to indicate the least difference. We also determined the correlation between SBPs at selected time points and the 24-hour mSBP using Pearson correlation (Table 1). Next, we analyzed the proportion of patients with SBPs measured at the selected time points that were within the range of $30 \%$ of 24 -hour mSBP using the Passing-Bablok regression method (Fig. 2) [19]. Then, we calculated the values of SBP at the selected time points that corresponded with the 24-hour SBP of $135 \mathrm{mmHg}$ using the Passing-Bablok regression equation. Using these calculated values of SBP and $135 \mathrm{mmHg}$ of the 24-hour SBP, we determined the uncontrolled hypertensive groups, which have a higher SBP than the calculated SBP or $135 \mathrm{mmHg}$. Uncontrolled hypertension was defined according to the suggestions of Obara et al. [20]. We compared the extent to which each uncontrolled hypertensive group that was identified based on
Table 1. Differences and correlations between systolic blood pressure (SBP) at each time point and the 24-hour mean SBP $^{\mathbf{a}}$

\begin{tabular}{lcc}
\hline Time point & $t^{\mathrm{b}}$ & $r^{\mathrm{c}}$ \\
\hline 7:00 AM & 0.241 & 0.683 \\
9:30 PM & 1.064 & 0.668 \\
2:00 PM & 3.696 & 0.521 \\
Office mean SBP & 12.380 & 0.532 \\
\hline
\end{tabular}

Three time points in order of decreasing differences from the 24-hour mean SBP and office mean SBP.

${ }^{\mathrm{b}}$ Difference between SBPs at each time point and 24-hour mean SBP by the paired $t$ test $(p>0.05)$.

${ }^{\mathrm{c}}$ Pearson correlation coefficient of SBPs at each time point and 24-hour mean SBP $(p<0.001)$.

the calculated BP at the selected time points was consistent with the hypertensive group based on $135 \mathrm{mmHg}$ by chi-square analysis. Finally, we calculated a receiver operating characteristic (ROC) curve for the diagnostic value of SBP at the selected time points in the diagnosis of uncontrolled hypertension. In all analyses, $p<0.05$ (two-sided) was taken to indicate statistical significance. Statistical analyses were performed using SPSS version 19 (IBM Co., Armonk, NY, USA) and R i386 version 3.o.o.

\section{RESULTS}

A total of 1,317 patients from 21 centers were enrolled 
in this study (Table 2). Their average age was $56.6 \pm 11.9$ years, and $62.9 \%$ were males. Most patients (48.6\%) were CKD stage 3, and the cause of CKD in almost $40 \%$ of the patients was hypertension. The office $\mathrm{mSBP}$ was $137 \pm 19.9 \mathrm{mmHg}$, and the 24 -hour $\mathrm{mSBP}$ was $131 \pm 16.4$

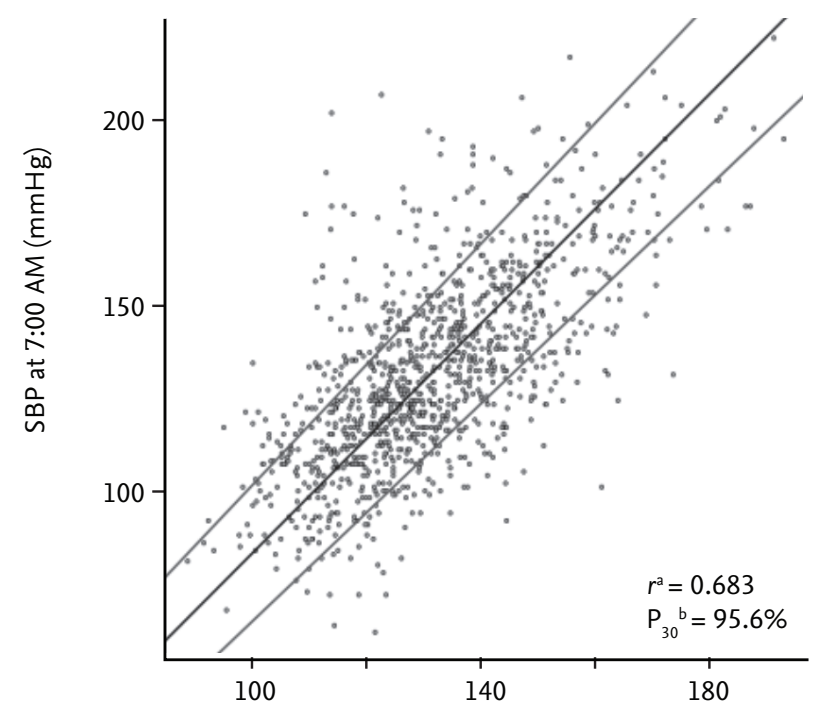

A 24-Hour $(\mathrm{mmHg})$

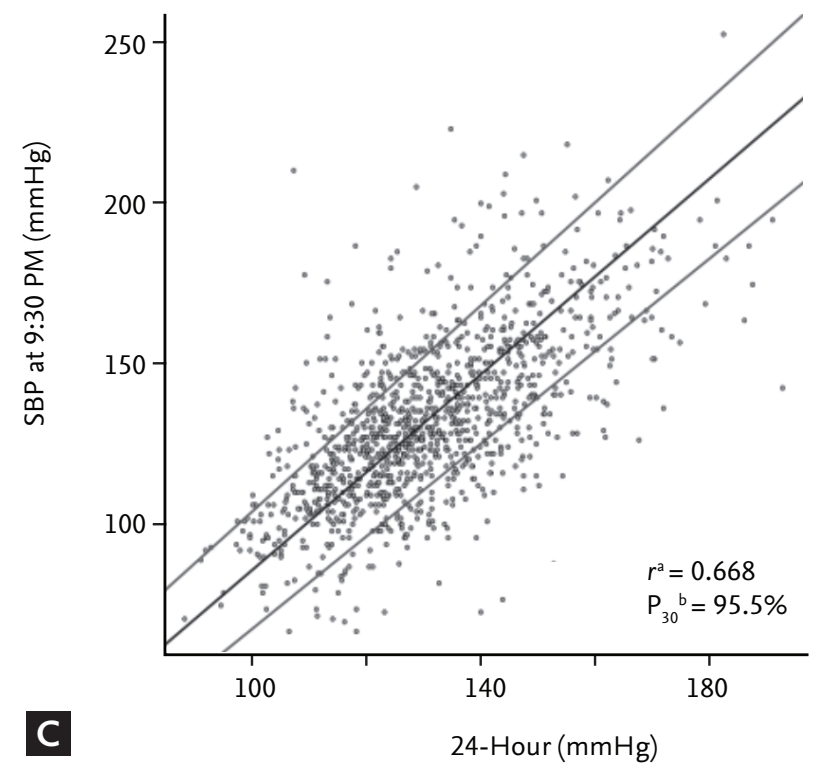

mmHg.

Fig. 1 shows the differences between 24-hour mSBP and the SBPs at each time point for 24 hours. Using the paired $t$ test ( $t$ value) inversely for the differences and correlations ( $r$ value) with the 24-hour mSBP, 7:00 AM,
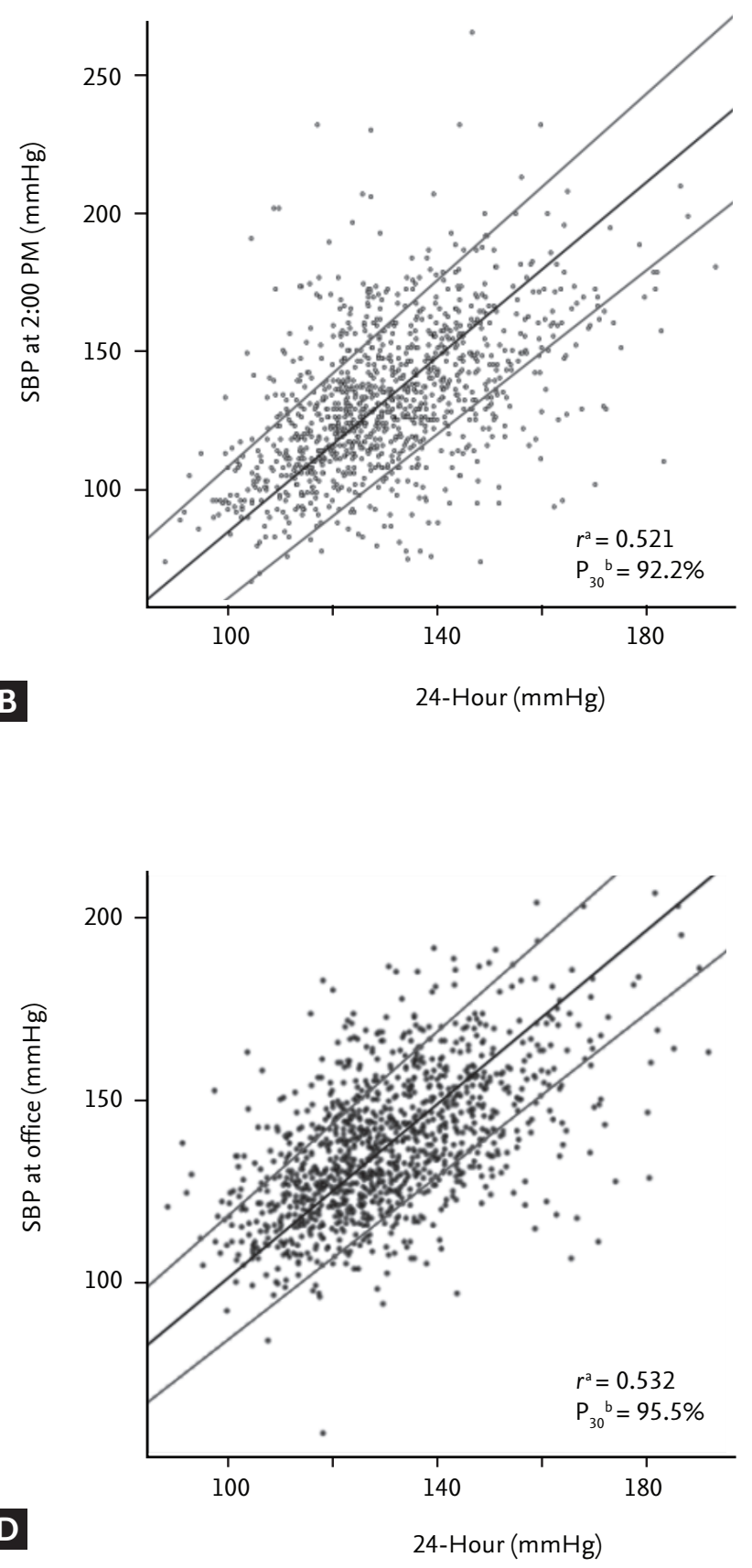

Figure 2. Correlations between systolic blood pressure (SBP) at specific time points and the 24-hour mean SBP. Associations between SBPs were analyzed at three specific time points (A, 7:00 AM; B, 2:00 PM; and C, 9:30 PM), office measurement (D, office mean SBP), and the 24-hour mean SBP by Passing-Bablok regression. All of the above parameters were statistically significant. aPearson correlation coefficient of SBP at each time point and the 24-hour mean SBP. bThe proportion of patients with SBPs within $30 \%$ of the 24 -hour mean SBP. 
Table 2. Baseline characteristics $(n=1,317)$

\begin{tabular}{lc}
\hline Clinical characteristic & Value \\
\hline Age, yr & $56.6 \pm 11.9$ \\
Body mass index, $\mathrm{kg} / \mathrm{m}^{2}$ & $25.3 \pm 3.42$ \\
\hline Duration of hypertension, mon & $99.4 \pm 88.8$ \\
eGFR $^{\mathrm{a}}, \mathrm{mL} / \mathrm{min} / 1.73 \mathrm{~m}^{2}$ & $48.8 \pm 19.4$ \\
\hline Proteinuria & $726(55.1)$ \\
\hline Office SBP, mmHg & $137 \pm 19.9$ \\
\hline $24-H o u r$ mean SBP, mmHg & $131 \pm 16.4$ \\
\hline Dipper & $588(44.6)$ \\
\hline Male sex & $828(62.9)$ \\
\hline Causes of chronic kidney disease & \\
\hline Hypertension & $510(38.7)$ \\
\hline Diabetes & $297(22.6)$ \\
\hline Chronic glomerulonephritis & $289(21.9)$ \\
\hline Others & $221(16.8)$ \\
\hline Current smoker & $196(14.9)$ \\
\hline Current alcohol consumer & $465(35.3)$ \\
\hline Chronic kidney disease stages & \\
\hline ACEi or ARB user & \\
\hline 3 & $416(31.6)$ \\
\hline
\end{tabular}

Values are presented as mean $\pm \mathrm{SD}$ or number (\%). eGFR, estimated glomerular filtration rate; SBP, systolic blood pressure; ACEi, angiotensin converting enzyme inhibitor; ARB, angiotensin receptor blocker.

a eGFR, estimated GFR calculated by MDRD.

${ }^{\mathrm{b}}$ Proteinuria, urine protein/creatinine ratio $(\mathrm{mg} / \mathrm{gCr}) \geq 300$.

${ }^{\mathrm{c}}$ Dipper, nighttime SBP/daytime SBP ratio < o.9.

${ }^{\mathrm{d}}$ Patient taking an ACEi or ARB, respectively.

2:00 PM, and 9:30 PM were chosen according to the order of decreasing difference from 24-hour mSBP. Thus, 7:00 AM, 2:00 PM, and 9:30 PM were considered significant time points at which the SBP was most closely correlated with the 24 -hour $\mathrm{mSBP}$ (Table 1). The largest difference was 20.299 at 3:30 AM.

The linear correlation between the SBPs at three specific time points, the office mSBP, and the 24-hour $\mathrm{mSBP}$ based on the Passing-Bablok regression is shown in Fig. 2. In this figure, the SBPs at 7:00 AM and 9:30 PM (Fig. 2A and 2C) showed the best correlations with the 24-hour mSBP, compared with measurement at 2:00 PM and the office SBP (Fig. $2 \mathrm{~B}$ and $2 \mathrm{D}$ ). The same re-
Table 3. Values of systolic blood pressure (SBP) at each time point corresponding to the 24-hour mean SBP of 135 mmHg based on the Passing-Bablok regression equation

\begin{tabular}{lc}
\hline Parameter & Cut-off value \\
\hline 7:00 AM & 136.74 \\
2:00 PM & 140.13 \\
9:30 PM & 138.78 \\
Office mean SBP & 142.61 \\
\hline
\end{tabular}

gression method showed that the highest proportion of patients with SBP measurements within 30\% of the 24-hour mSBP (proportion 30, $\mathrm{P}_{30}$ ) were obtained at 7:00 $\mathrm{AM}$ and 9:30 $\mathrm{PM}\left(\mathrm{P}_{30}=95.6 \%, \mathrm{P}_{30}=95.3 \%\right.$, respectively $)$. At 2:00 PM, $92.1 \%$ of the patients were included within $\mathrm{P}_{30}$ (Fig. 2).

Using the Passing-Bablok equation, we calculated the SBPs at the three time points as values that corresponded with the 24-hour mSBP of $135 \mathrm{mmHg}$ (Table 3). Measurements at 7:00 AM and 9:30 PM were much closer to $135 \mathrm{mmHg}$ (136.74 and $138.78 \mathrm{mmHg}$, respectively). The office $\mathrm{mSBP}$ exhibited the greatest difference from $135 \mathrm{mmHg}$ (142.61 mmHg). Using these calculated SBPs at the three time points and the 24-hour mSBP of 135 $\mathrm{mmHg}$, the patients were classified into uncontrolled hypertensive groups, and the agreement among groups was analyzed (Fig. 3). The uncontrolled hypertensive group based on values at 7:00 AM and 9:30 PM showed the best agreement with the group at $\geq 135 \mathrm{mmHg}$ compared with the other groups (7:00 AM, 70.8\%; 9:30 PM, $68.1 \%$; $p<0.001$ ).

We evaluated the usefulness of the calculated SBP values at the three time points and the office measurement for diagnosis of uncontrolled hypertension with a ROC curve (Fig. 4). The ROC curve showed that 7:00 AM and 9:30 PM had markedly larger area under the curve (AUC) values $(\mathrm{AUC}=0.843, p<0.001 ; \mathrm{AUC}=0.831, p<0.001$, respectively). The AUC values at 7:00 AM and 9:30 PM were significantly greater than those at 2:00 PM and the office measurement.

We performed subgroup analyses to test whether these time points for BP measurement were clinically meaningful. In CKD stage 2 patients, the SBPs at 7:30 AM, 3:00 PM, and 10:00 PM had smaller differences than those at previous time points; however, in the correlation or $\mathrm{P}_{30}$ analyses, new time points showed similar or lower 


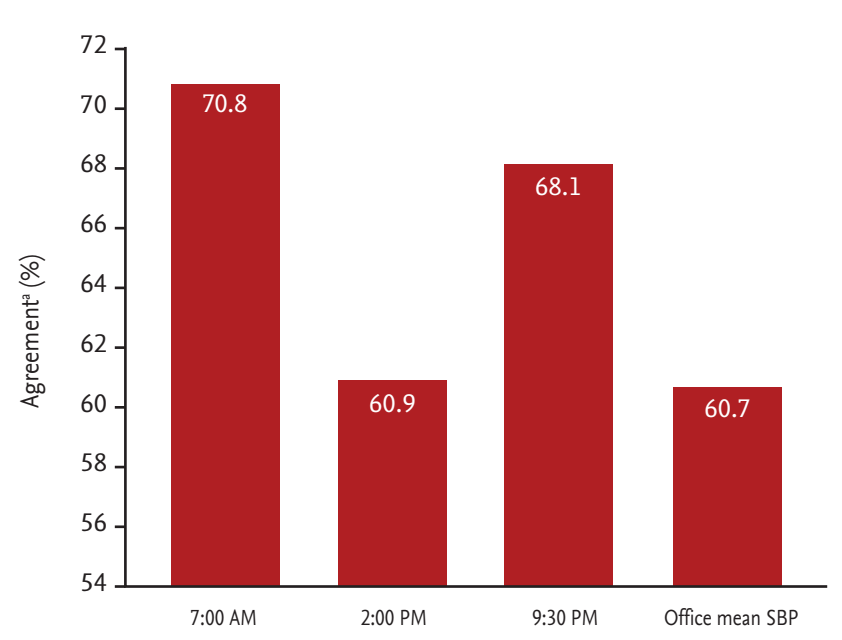

Figure 3. Agreement between uncontrolled hypertensive groups based on the calculated systolic blood pressure (SBP) at each time point and the 24-hour mean SBP of $135 \mathrm{mmHg}$. ${ }^{a}$ Patients were classified into uncontrolled hypertensive groups based on the 24 -hour SBP of $135 \mathrm{mmHg}$ and the SBP values corresponding to $135 \mathrm{mmHg}$ were calculated at the three selected time points along with the office measurement (7:00 AM, 136.74 mmHg; 2:00 PM, 140.13 mmHg; 9:30 $\mathrm{PM}, 138.78 \mathrm{mmHg}$; office mean SBP, $142.61 \mathrm{mmHg}$ ). Then, we analyzed the agreement (\%) between the uncontrolled hypertension groups using the chi-square test.

values compared with the three previously selected time points (7:00 AM, 2:00 PM, and 9:30 PM) (Table 4). In CKD stage 4 patients, the SBP measurements at 6:30 PM and 3:00 PM were closer to the 24-hour mSBP, but the SBP at 7:00 AM showed a better correlation with the 24-hour $\mathrm{mSBP}$ than the SBP at 6:30 PM, and more patients were included in $\mathrm{P}_{30}$ at 2:00 PM than at 3:00 PM.

The patients were classified as dippers if they had a ratio of $<0.9$ and non-dippers if they had a ratio of $\geq 0.9$. The dipper patients showed the same results as all patients, but in the non-dipper patients SBPs at 4:30 AM, 4:30 PM, and 11:00 PM had lower differences than at the three previous specific time points. However, based on the correlation and $\mathrm{P}_{30}$ analysis, the values at 7:00 AM, 2:00 PM, and 9:30 PM were found to be superior to the new time points (data not shown).

We also analyzed the subgroups by daytime and nighttime. The standard time points for classification into day and night were 8:00 AM and 10:00 PM, respectively, so the findings were different from the previous results. In daytime, the SBPs at 9:00 AM and 1:30 PM correlated with daytime $\mathrm{mSBP}$, and at nighttime, the SBP at 4:30

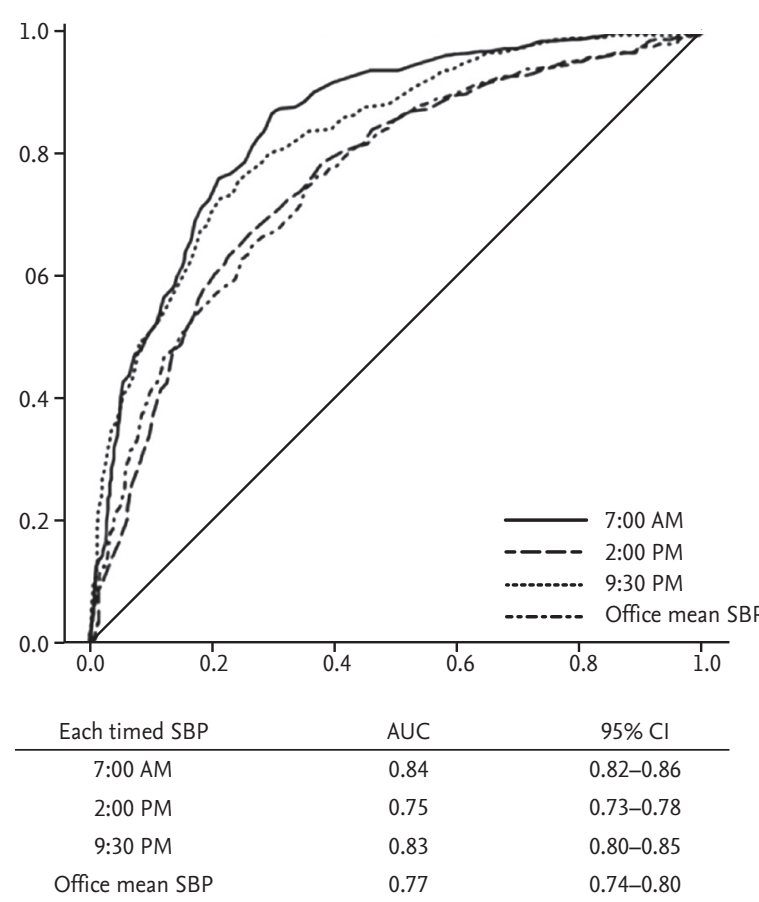

Figure 4. Receiver operating characteristic curves between the 24-hour mean systolic blood pressure (SBP) and 7:00 AM, 2:00 PM, and 9:30 PM and the office mean SBP for diagnosis of uncontrolled hypertension. The area under the curve (AUC) values of 7:00 AM and 9:30 PM were significantly higher than those of 2:00 PM and the office mean SBP based on DeLong's test. CI, confidence interval.

AM correlated with nighttime mSBP (data not shown).

In other subgroups, such as diabetes/non-diabetes, left ventricular hypertrophy (LVH)/non-LVH, proteinuria/non-proteinuria, and gender groups, results similar to those for all patients were obtained using identical analysis methods. In these groups, predominantly the SBPs at 7:00 AM and 9:30 PM were highly correlated with the 24-hour mSBP (data not shown).

\section{DISCUSSION}

We examined the time points at which the measured SBP showed the closest correlation with the 24-hour $\mathrm{mSBP}$ in the hope that SBP measurements at those time points could replace the 24 -hour mSBP in CKD patients. Out-of-office BP (ABPM, HBPM) provides different information about the patient's BP condition, and so cannot be considered to be equivalent or alternative methods [6]. HBPM is reproducible and more convenient 
Table 4. Correlation and proportion 30 between the 24-hour mean systolic blood pressure (SBP) and the SBP at specific time points according to chronic kidney disease stage

\begin{tabular}{|c|c|c|c|c|c|c|c|c|}
\hline \multirow{2}{*}{ Time point } & \multicolumn{2}{|c|}{ Total } & \multicolumn{2}{|c|}{ Stage 2} & \multicolumn{2}{|c|}{ Stage 3} & \multicolumn{2}{|c|}{ Stage 4} \\
\hline & $r^{\mathrm{a}}$ & $\mathrm{P}_{30}, \%^{\mathrm{b}}$ & $r^{\mathrm{a}}$ & $\mathrm{P}_{30}, \%{ }^{\mathrm{b}}$ & $r^{\mathrm{a}}$ & $\mathrm{P}_{30}, \%^{\mathrm{b}}$ & $r^{\mathrm{a}}$ & $\mathrm{P}_{30}, \%^{\mathrm{b}}$ \\
\hline 6:30 PM & & & & & & & 0.746 & 96.4 \\
\hline 7:00 AM & 0.683 & $95 \cdot 4$ & 0.576 & $95 \cdot 3$ & 0.678 & 95.8 & 0.762 & 94.7 \\
\hline $7: 30 \mathrm{AM}$ & & & 0.529 & 92.5 & & & & \\
\hline 2:00 PM & 0.521 & 92.2 & 0.524 & 91.8 & 0.521 & 91.3 & 0.567 & 94.8 \\
\hline 3:00 PM & & & 0.580 & 91.8 & & & 0.596 & 94.2 \\
\hline 9:30 PM & 0.668 & $95 \cdot 5$ & 0.665 & 95.0 & 0.645 & $95 \cdot 4$ & 0.708 & 96.4 \\
\hline 10:00 PM & & & 0.651 & $94 \cdot 3$ & & & & \\
\hline Office mean SBP & 0.532 & $95 \cdot 5$ & 0.505 & 96.6 & 0.500 & 95.0 & 0.592 & 95.0 \\
\hline
\end{tabular}

$\mathrm{P}_{30}$, proportion 30.

${ }^{a}$ Pearson correlation coefficient of SBP at each time point and 24-hour mean SBP.

${ }^{b}$ Proportion of patients included within $30 \%$ of the mean SBP for 24 hours according to the Passing-Bablok method. All of the above parameters were statistically significant $(p<0.001)$.

than ABPM, but few studies have examined the detailed guidelines for the specific time points for self-BP measurement, especially in CKD patients [9,21,22]. Here, we examined whether there may be a specific time of the day for measuring BP that is representative of the mean BP over the entire day and attempted to identify a specific time point for such BP measurement. The present study is among the first to suggest specific time points for out-of-office BP measurement in CKD patients.

We used several statistical methods to verify these specific time points for BP measurement. The results of several analyses consistently showed that measurements at three specific time points; i.e., 7:00 AM, 2:00 PM, and 9:30 PM, were closely correlated with the 24-hour mSBP. Therefore, we considered that these could be used as specific time points in the morning, afternoon, and night, respectively. Among them, the SBPs at 7:00 AM and 9:30 PM were more closely correlated with the 24hour mSBP than that at 2:00 PM or office mSBP. These findings also indicated that the office $\mathrm{mSBP}$ is inferior to SBPs at our selected time points. The weaker correlation of the 2:00 PM value may be due to variations in BP [23]. The BP at 2:00 PM can be affected by multiple factors, such as stress, diet, physical condition, smoking, and caffeine intake, although the difference between BP at 2:00 PM and the 24-hour mSBP was minimal. In addition, office $\mathrm{mSBP}$ may be influenced by the white coat effect.

In subgroup analysis, we obtained slightly different results in patients with CKD stage 2 and CKD stage 4 , as well as the non-dippers. In the CKD stage 4 and non-dipper groups, these differences may be explained by the variability in SBP according to progression of renal disease. Patients with advanced stage CKD have a tendency toward variable BP and the dipper phenomenon [24]. The different agreement between the uncontrolled hypertensive groups with CKD stage 4 could be explained in the same way. However, the different time points in these subgroups were mostly included within approximately the same time ranges, such as 7:00 $\mathrm{AM}$, 2:00 PM, and 9:30 PM. In addition, the ROC curve supported these results in all of the subgroups. In the non-dipper and day/nighttime groups, we obtained findings that differed from the previous results. In the non-dipper group, the 4:30 AM, 4:30 PM, and 11:00 PM time points differed less from the 24 -hour mSBP, but regression analyses showed that the correlations of the three previous time points with the 24 -hour mSBP were higher than the newly presented time points. Therefore, 7:00 AM, 2:00 PM, and 9:30 PM were considered not to be inferior to the new time points in terms of correlation with the 24-hour mSBP. In the day/nighttime groups, 9:00 AM and 1:30 PM in the daytime group and 4:30 AM in the nighttime group were correlated with the daytime 
and nighttime mSBP. These results may have been influenced by the different time ranges in the daytime and nighttime designations of 8:00 AM and 10:00 PM. In addition, it is possible that several factors, such as physical activity and diet, influenced the daytime results. According to these results, we recommend that patients check their BP at 4:30 AM for the nighttime BP, but this time point is inconvenient for clinical use. Therefore, we suggest time points that are more convenient and clinically useful: checking BP at 7:00 AM, 2:00 PM, and 9:30 PM for whole-day monitoring would better ensure patient compliance.

The strength of the present study is that it suggested specific time points for out-of-office BP measurement, and involved CKD patients. Most studies of out-of-office BP schedules have been performed in populations with normal renal function $[9,10,25,26]$. A previous study on HBPM in CKD patients showed that out-of-office BP was better than office BP for diagnosis of hypertension; however, it did not specify the timing of the BP measurements [27]. By using the SBP values at these specific time points, we could expect to monitor the BP status of patients more effectively, because the SBPs at the three time points were better correlated to the 24 -hour $\mathrm{mSBP}$ than SBPs at other time points. We suggest the use of these time points for general monitoring of patient BP status rather than a diagnostic or prognostic method. For prognosis, nighttime BP measurement or ABPM should also be performed.

This study had several limitations. We checked ABPM only once, so between-visit variation in SBP could not be evaluated. Some studies restricted the times for BP measurement considering diet, sleep, and medication, while we did not because the purpose of this study was to determine more clinically convenient time points for BP measurement to increase patient compliance. We excluded DBP from these analyses to avoid correlations of a large number of time points for each SBP and DBP with the 24-hour mean BP. To verify the effects of these time points in monitoring and prognosis, future studies should consider several factors that influence BP and should include both SBP and DBP.

In conclusion, we found that 7:00 AM and 9:30 PM were specific time points that were highly correlated with the 24-hour mSBP for out-of-office SBP measurement in CKD patients. According to several analytical methods, these time points showed consistent correlations with 24-hour mSBP overall. Although further studies are needed to verify the practical usefulness and prognostic value of these results, this study represents a valuable starting point in the effort to establish more specific time points for SBP measurement.

\section{KEY MESSAGE}

1. Ambulatory blood pressure (BP) monitoring has been recommended for evaluating the status of $\mathrm{BP}$ control, but its practical use is difficult.

2. We postulated that some specific time points may be well correlated with, and could be used as representatives of, the 24-hour mean systolic blood pressure (SBP) in hypertensive chronic kidney disease patients.

3. We found that the SBPs at 7:00 AM and 9:30 PM were well correlated with 24-hour mean SBP, and therefore checking blood pressure at these specific time points could be important for monitoring of blood pressure control in patients.

\section{Conflict of interest}

This work was sponsored by Sanofi Korea Co., Ltd.

\section{Acknowledgments}

A special acknowledgment is extended to the Medical Research Collaborating Center of Seoul National University Bundang Hospital and APrODiTe study participants for their time and commitment. The authors are indebted to J. Patrick Barron, Professor Emeritus, Tokyo Medical University and Adjunct Professor, Seoul National University Bundang Hospital, for his pro bono editing of this manuscript.

\section{REFERENCES}

1. Clement DL, De Buyzere ML, De Bacquer DA, et al. Prognostic value of ambulatory blood-pressure recordings in patients with treated hypertension. N Engl J Med 2003;348:2407-2415.

2. Agarwal R, Andersen MJ. Correlates of systolic hyperten- 
sion in patients with chronic kidney disease. Hypertension 2005;46:514-520.

3. Hanratty R, Chonchol M, Havranek EP, et al. Relationship between blood pressure and incident chronic kidney disease in hypertensive patients. Clin J Am Soc Nephrol 2011;6:2605-2611.

4. Gabbai FB, Rahman M, Hu B, et al. Relationship between ambulatory BP and clinical outcomes in patients with hypertensive CKD. Clin J Am Soc Nephrol 2012;7:1770-1776.

5. Jin DC. Current status of dialysis therapy in Korea. Korean J Intern Med 2011;26:123-131.

6. Mancia G, Fagard R, Narkiewicz K, et al. 2013 ESH/ESC Guidelines for the management of arterial hypertension: the Task Force for the management of arterial hypertension of the European Society of Hypertension (ESH) and of the European Society of Cardiology (ESC). J Hypertens 2013;31:1281-1357.

7. Omvik P, Gerhardsen G. The Norwegian office-, home-, and ambulatory blood pressure study (NOHA). Blood Press 2003;12:211-219.

8. Pickering TG, Hall JE, Appel LJ, et al. Recommendations for blood pressure measurement in humans and experimental animals: Part 1: blood pressure measurement in humans: a statement for professionals from the Subcommittee of Professional and Public Education of the American Heart Association Council on High Blood Pressure Research. Hypertension 2005;45:142-161.

9. Pickering TG, Miller NH, Ogedegbe G, et al. Call to action on use and reimbursement for home blood pressure monitoring: executive summary: a joint scientific statement from the American Heart Association, American Society Of Hypertension, and Preventive Cardiovascular Nurses Association. Hypertension 2008;52:1-9.

10. Verberk WJ, Kroon AA, Kessels AG, de Leeuw PW. Home blood pressure measurement: a systematic review. J Am Coll Cardiol 2005;46:743-751.

11. Alborzi P, Patel N, Agarwal R. Home blood pressures are of greater prognostic value than hemodialysis unit recordings. Clin J Am Soc Nephrol 2007;2:1228-1234.

12. Parati G, Stergiou GS, Asmar R, et al. European Society of Hypertension guidelines for blood pressure monitoring at home: a summary report of the Second International Consensus Conference on Home Blood Pressure Monitoring. J Hypertens 2008;26:1505-1526.

13. Ogihara T, Kikuchi K, Matsuoka H, et al. The Japanese Society of Hypertension guidelines for the management of hypertension (JSH 2009). Hypertens Res 2009;32:3-107.

14. Cha RH, Kim S, Ae Yoon S, et al. Association between blood pressure and target organ damage in patients with chronic kidney disease and hypertension: results of the APrODiTe study. Hypertens Res 2014;37:172-178.

15. Fagard R, Brguljan J, Thijs L, Staessen J. Prediction of the actual awake and asleep blood pressures by various methods of $24 \mathrm{~h}$ pressure analysis. J Hypertens 1996;14:557-563.

16. Ohkubo T, Imai Y, Tsuji I, et al. Home blood pressure measurement has a stronger predictive power for mortality than does screening blood pressure measurement: a population-based observation in Ohasama, Japan. J Hypertens 1998;16:971-975.

17. Hozawa A, Ohkubo T, Nagai K, et al. Prognosis of isolated systolic and isolated diastolic hypertension as assessed by self-measurement of blood pressure at home: the Ohasama study. Arch Intern Med 2000;160:3301-3306.

18. Bland JM, Altman DG. Statistical methods for assessing agreement between two methods of clinical measurement. Lancet 1986;1:307-310.

19. Passing H, Bablok. A new biometrical procedure for testing the equality of measurements from two different analytical methods: application of linear regression procedures for method comparison studies in clinical chemistry, part I. J Clin Chem Clin Biochem 1983;21:709-720.

20. Obara T, Ohkubo T, Funahashi J, et al. Isolated uncontrolled hypertension at home and in the office among treated hypertensive patients from the J-HOME study. J Hypertens 2005;23:1653-1660.

21. Stergiou GS, Skeva II, Zourbaki AS, Mountokalakis TD. Self-monitoring of blood pressure at home: how many measurements are needed? J Hypertens 1998;16:725-731.

22. Stergiou GS, Parati G. The optimal schedule for self-monitoring of blood pressure by patients at home. J Hypertens 2007;25:1992-1997.

23. Agarwal R, Light RP. Physical activity and hemodynamic reactivity in chronic kidney disease. Clin J Am Soc Nephrol 2008;3:1660-1668.

24. Singh S. Etiology and management of hypertension in chronic kidney disease. Clin Queries Nephrol 2012;1:259267.

25. Imai Y, Nishiyama A, Sekino M, et al. Characteristics of blood pressure measured at home in the morning and in the evening: the Ohasama study. J Hypertens 1999;17:889898.

26. Ohkubo T, Asayama K, Kikuya M, et al. How many times 
should blood pressure be measured at home for better prediction of stroke risk? Ten-year follow-up results from the Ohasama study. J Hypertens 2004;22:1099-1104.
27. Andersen MJ, Khawandi W, Agarwal R. Home blood pressure monitoring in CKD. Am J Kidney Dis 2005;45:9941001. 